\title{
Metric Dimension in Fuzzy Graphs and Neutrosophic Graphs
}

\author{
Henry Garrett
}

Independent Researcher

DrHenryGarrett@gmail.com

Twitter's ID: @DrHenryGarrett | @DrHenryGarrett.wordpress.com

\begin{abstract}
In this study, the term dimension is introduced on fuzzy graphs and neutrosophic graphs. The classes of these specific graphs are chosen to obtain some results based on dimension. The types of crisp notions and fuzzy notions are used to make sense about the material of this study and the outline of this study uses some new notions which are crisp and fuzzy.
\end{abstract}

Keywords: Fuzzy Graphs, Neutrosophic Graphs, Dimension

AMS Subject Classification: 05C17, 05C22, 05E45

\section{Background}

Some literatures like cardinality of set, n-set, notation of fuzzy graphs, common vertex set with same values for vertices and edges, permutation, fuzzy vertex set: vertices and their values, fuzzy edge set: edges and their values. fixed-edge fuzzy graphs fixed-vertex fuzzy graphs, fuzzy twin vertices are used. For using material look at [1-15].

\section{Definitions}

We use the notion of vertex in fuzzy graphs to define new notions which state the relation amid vertices. In this way, the set of vertices are distinguished by another set of vertices.

Definition 2.1. Let $G=(V, \sigma, \mu)$ be a fuzzy graph. A vertex $m$ fuzzy-resolves vertices $f_{1}$ and $f_{2}$ if $d\left(m, f_{1}\right) \neq d\left(m, f_{2}\right)$. A set $M$ is fuzzy resolving set if for every couple of vertices $f_{1}, f_{2} \in V \backslash M$, there's a vertex $m \in M$ such that $m$ fuzzy-resolves $f_{1}$ and $f_{2}$. $|M|$ is called fuzzy metric number of $G$ and $\min _{M} \Sigma_{m \in M} \sigma(m)$ is called fuzzy metric dimension of $G$ and if fuzzy metric number of set $M$ equals fuzzy metric dimension, then $M$ is called fuzzy metric set of $G$.

Example 2.2. Let $G$ be a fuzzy graph as figure (1). By applying Table (1), the 1-set is explored which its cardinality is minimum. $\left\{f_{6}\right\}$ and $\left\{f_{4}\right\}$ are 1 -set which has minimum cardinality amid all sets of vertices but $\left\{f_{4}\right\}$ isn't fuzzy resolving set and $\left\{f_{6}\right\}$ is fuzzy resolving set. Thus there's no fuzzy metric set but $\left\{f_{6}\right\} . f_{6}$ fuzzy-resolves all given couple of vertices. Therefore one is fuzzy metric number of $G$ and 0.13 is fuzzy metric 


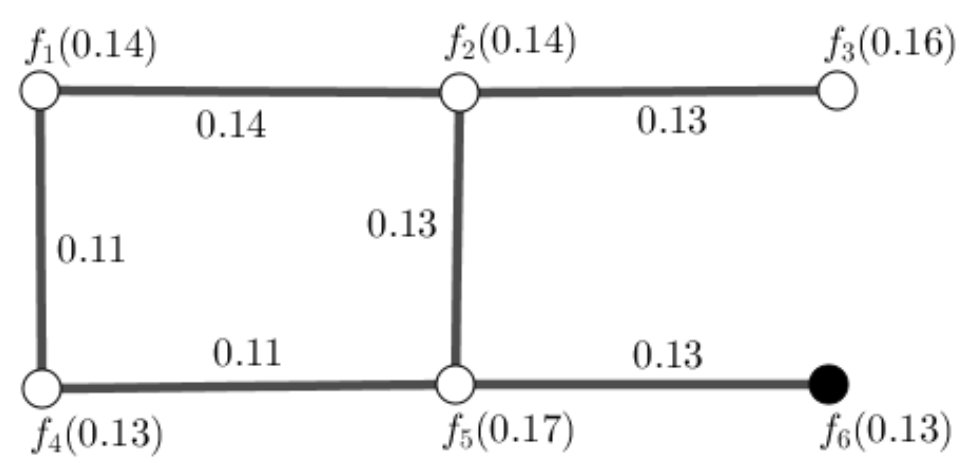

Figure 1. Black vertex $\left\{f_{6}\right\}$ is only fuzzy metric set amid all sets of vertices for fuzzy graph $G$.

dimension of $G$. By using Table (1), $f_{4}$ doesn't fuzzy-resolve $f_{2}$ and $f_{6} . f_{4}$ doesn't fuzzy-resolve $f_{1}$ and $f_{5}$, too.

Table 1. Distances of Vertices from sets of vertices $\left\{f_{6}\right\}$ and $\left\{f_{4}\right\}$ in Fuzzy Graph $G$.

\begin{tabular}{c|lccccc}
\hline Vertices & $f_{1}$ & $f_{2}$ & $f_{3}$ & $f_{4}$ & $f_{5}$ & $f_{6}$ \\
\hline$f_{6}$ & 0.22 & 0.26 & 0.39 & 0.24 & 0.13 & 0 \\
Vertices & $f_{1}$ & $f_{2}$ & $f_{3}$ & $f_{4}$ & $f_{5}$ & $f_{6}$ \\
$f_{4}$ & 0.11 & 0.24 & 0.37 & 0 & 0.11 & 0.24 \\
\hline
\end{tabular}

Definition 2.3. Consider $\mathcal{G}$ as a family of fuzzy graphs on a common vertex set $V$. A vertex $m$ simultaneously fuzzy-resolves vertices $f_{1}$ and $f_{2}$ if $d_{G}\left(m, f_{1}\right) \neq d_{G}\left(m, f_{2}\right)$, for all $G \in \mathcal{G}$. A set $M$ is simultaneously fuzzy resolving set if for every couple of vertices $f_{1}, f_{2} \in V \backslash M$, there's a vertex $m \in M$ such that $m$ resolves $f_{1}$ and $f_{2}$, for all $G \in \mathcal{G}$. $|M|$ is called simultaneously fuzzy metric number of $\mathcal{G}$ and $\min \sigma_{m \in V} \sigma(m)$ is called simultaneously fuzzy metric dimension of $\mathcal{G}$ and if the simultaneously fuzzy cardinality of set $M$ equals simultaneously fuzzy metric dimension, then $M$ is called simultaneously fuzzy metric set of $\mathcal{G}$.

Example 2.4. Let $\mathcal{G}=\left\{G_{1}, G_{2}, G_{3}\right\}$ be a collection of fuzzy graphs with common fuzzy vertex set and a subset of fuzzy edge set as figure (2). By applying Table (2), the 1 -set is explored which its cardinality is minimum. $\left\{f_{2}\right\}$ and $\left\{f_{4}\right\}$ are 1 -set which has minimum cardinality amid all sets of vertices. $\left\{f_{4}\right\}$ is as fuzzy resolving set as $\left\{f_{6}\right\}$ is. Thus there's no fuzzy metric set but $\left\{f_{4}\right\}$ and $\left\{f_{6}\right\} . f_{6}$ as fuzzy-resolves all given couple of vertices as $f_{4}$. Therefore one is fuzzy metric number of $\mathcal{G}$ and 0.13 is fuzzy metric dimension of $\mathcal{G}$. By using Table (2), $f_{4}$ fuzzy-resolves all given couple of vertices.

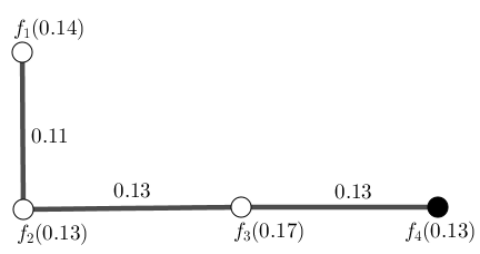

$G_{1}$

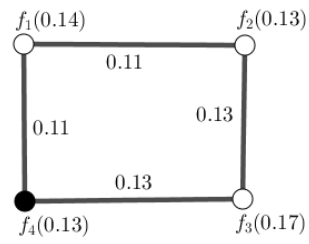

$G_{2}$

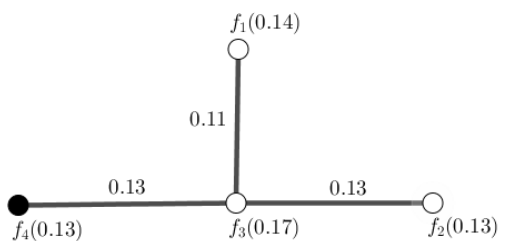

$G_{3}$

Figure 2. Black vertex $\left\{f_{4}\right\}$ and the set of vertices $\left\{f_{2}\right\}$ are simultaneously fuzzy metric set amid all sets of vertices for family of fuzzy graphs $\mathcal{G}$. 
Table 2. Distances of Vertices from set of vertices $\left\{f_{6}\right\}$ in Family of Fuzzy Graphs $\mathcal{G}$.

\begin{tabular}{c|lccc}
\hline Vertices of $G_{1}$ & $f_{1}$ & $f_{2}$ & $f_{3}$ & $f_{4}$ \\
\hline$f_{4}$ & 0.37 & 0.26 & 0.13 & 0 \\
Vertices of $G_{2}$ & $f_{1}$ & $f_{2}$ & $f_{3}$ & $f_{4}$ \\
$f_{4}$ & 0.11 & 0.22 & 0.13 & 0 \\
Vertices of $G_{3}$ & $f_{1}$ & $f_{2}$ & $f_{3}$ & $f_{4}$ \\
$f_{4}$ & 0.24 & 0.26 & 0.13 & 0 \\
\hline
\end{tabular}

\section{General Relationships}

Proposition 3.1. Let $G$ be a path fuzzy graph. Then every leaf is fuzzy resolving set.

Proof. Let $l$ be a leaf. For every given a couple of vertices $f_{i}$ and $f_{j}$, we get $d\left(l, f_{i}\right) \neq d\left(l, f_{j}\right)$. Since if we reassign indexes to vertices such that every vertex $f_{i}$ and $l$ have $i$ vertices amid themselves, then $d\left(l, f_{i}\right)=\Sigma_{j \leq i} \mu\left(f_{j} f_{i}\right) \leq i$. Thus $j \leq i$ implies

$\Sigma_{t \leq j} \mu\left(f_{t} f_{j}\right)+\Sigma_{j \leq s \leq i} \mu\left(f_{s} f_{i}\right)>\Sigma_{j \leq i} \mu\left(f f_{i}\right) \equiv d\left(l, f_{j}\right)+c=d\left(l, f_{i}\right) \equiv d\left(l, f_{j}\right)<d\left(l, f_{i}\right)$.

Therefore, by $d\left(l, f_{j}\right)<d\left(l, f_{i}\right)$, we get $d\left(l, f_{i}\right) \neq d\left(l, f_{j}\right) . f_{i}$ and $f_{j}$ are arbitrary so $l$ fuzzy-resolves any given couple of vertices $f_{i}$ and $f_{j}$ which implies $\{l\}$ is a fuzzy resolving set.

Corollary 3.2. Let $G$ be a fixed-edge path fuzzy graph. Then every leaf is fuzzy resolving set.

Proof. Let $l$ be a leaf. For every given couple of vertices, $f_{i}$ and $f_{j}$, we get $d\left(l, f_{i}\right)=c i \neq d\left(l, f_{j}\right)=c j$. It implies $l$ fuzzy-resolves any given couple of vertices $f_{i}$ and $f_{j}$ which implies $\{l\}$ is a fuzzy resolving set.

Corollary 3.3. Let $G$ be a fixed-vertex path fuzzy graph. Then every leaf is fuzzy metric set, fuzzy metric number is one and fuzzy metric dimension is c where $c=\sigma(f), f \in V$.

Proof. By Proposition (3.1), every leaf is fuzzy resolving set. By $c=\sigma(f), \forall f \in V$, every leaf is fuzzy metric set, fuzzy metric number is one and fuzzy metric dimension is $c$.

Proposition 3.4. Let $G$ be a path fuzzy graph. Then a set including every couple of vertices is fuzzy resolving set.

Proof. Let $f$ and $f^{\prime}$ be a couple of vertices. For every given a couple of vertices $f_{i}$ and $f_{j}$, we get either $d\left(f, f_{i}\right) \neq d\left(f, f_{j}\right)$ or $d\left(f^{\prime}, f_{i}\right) \neq d\left(f^{\prime}, f_{j}\right)$.

Corollary 3.5. Let $G$ be a fixed-edge path fuzzy graph. Then every set containing couple of vertices is fuzzy resolving set.

Proposition 3.6. Let $G$ be a fuzzy graph. An $(k-1)$-set from an $k$-set of fuzzy twin vertices is subset of a fuzzy resolving set.

Proof. If $t$ and $t^{\prime}$ are fuzzy twin vertices, then $N(t)=N\left(t^{\prime}\right)$ and $\mu(t s)=\mu\left(t^{\prime} s\right)$, for all $s \in N(t)=N\left(t^{\prime}\right)$.

Corollary 3.7. Let $G$ be a fuzzy graph. The number of fuzzy twin vertices is $n-1$. Then fuzzy metric number is $n-2$.

Corollary 3.8. Let $G$ be a fuzzy graph. The number of fuzzy twin vertices is $n-1$. Then $G$ is fixed-edge fuzzy graph. 
Corollary 3.9. Let $G$ be a fixed-vertex fuzzy graph. The number of fuzzy twin vertices ${ }_{67}$ is $n-1$. Then fuzzy metric number is $n-2$, fuzzy metric dimension is $(n-2) \sigma(m)$ where $m$ is fuzzy twin vertex with a vertex. Every $(n-2)$-set including fuzzy twin vertices is fuzzy metric set.

Proposition 3.10. Let $G$ be a fixed-vertex fuzzy graph such that it's fuzzy complete. Then fuzzy metric number is $n-1$, fuzzy metric dimension is $(n-1) \sigma(m)$ where $m$ is a given vertex. Every $(n-1)$-set is fuzzy metric set.

Proof. In complete graph, every couple of vertices are twin vertices. By $G$ is a fixed-vertex fuzzy graph and it's fuzzy complete, every couple of vertices are fuzzy twin vertices. Thus by Proposition (3.6), the result follows.

Proposition 3.11. Let $\mathcal{G}$ be a family of fuzzy graphs with common vertex set. Then simultaneously fuzzy metric number of $\mathcal{G}$ is $n-1$.

Proof. Consider $(n-1)$-set. Thus there's no couple of vertices to be fuzzy-resolved. Therefore, every $(n-1)$-set is fuzzy resolving set for any given fuzzy graph. Then it holds for any fuzzy graph. It implies it's fuzzy resolving set and its cardinality is fuzzy metric number. $(n-1)$-set has the cardinality $n-1$. Then it holds for any fuzzy graph. It induces it's simultaneously fuzzy resolving set and its cardinality is simultaneously fuzzy metric number.

Proposition 3.12. Let $\mathcal{G}$ be a family of fuzzy graphs with common vertex set. Then simultaneously fuzzy metric number of $\mathcal{G}$ is greater than the maximum fuzzy metric number of $G \in \mathcal{G}$.

Proof. Suppose $t$ and $t^{\prime}$ are simultaneously fuzzy metric number of $\mathcal{G}$ and fuzzy metric number of $G \in \mathcal{G}$.. Thus $t$ is fuzzy metric number for any $G \in \mathcal{G}$. Hence, $t \geq t^{\prime}$. So simultaneously fuzzy metric number of $\mathcal{G}$ is greater than the maximum fuzzy metric number of $G \in \mathcal{G}$.

Proposition 3.13. Let $\mathcal{G}$ be a family of fuzzy graphs with common vertex set. Then simultaneously fuzzy metric number of $\mathcal{G}$ is greater than simultaneously fuzzy metric number of $\mathcal{H} \subseteq \mathcal{G}$.

Proof. Suppose $t$ and $t^{\prime}$ are simultaneously fuzzy metric number of $\mathcal{G}$ and $\mathcal{H}$. Thus $t$ is fuzzy metric number for any $G \in \mathcal{G}$. It implies Thus $t$ is fuzzy metric number for any $G \in \mathcal{H}$. So $t$ is simultaneously fuzzy metric number of $\mathcal{H}$. By applying Definition about being the minimum number, $t \geq t^{\prime}$. So simultaneously fuzzy metric number of $\mathcal{G}$ is greater than simultaneously fuzzy metric number of $\mathcal{H} \subseteq \mathcal{G}$.

Theorem 3.14. Fuzzy twin vertices aren't resolved in any given fuzzy graph.

Proof. Let $t$ and $t^{\prime}$ are fuzzy twin vertices. Then $N(t)=N\left(t^{\prime}\right)$ and $\mu(t s)=\mu\left(t^{\prime} s\right)$, for all edges $t s, t^{\prime} s \in E$. Thus for every given vertex $s^{\prime} \in V, d_{G}\left(s^{\prime}, t\right)=d_{G}(s, t)$ where $G$ is a given fuzzy graph. It means that $t$ and $t^{\prime}$ aren't resolved in any given fuzzy graph. $t$ and $t^{\prime}$ are arbitrary so fuzzy twin vertices aren't resolved in any given fuzzy graph.

Proposition 3.15. Let $G$ be a fixed-vertex fuzzy graph. If $G$ is fuzzy complete, then every couple of vertices are fuzzy twin vertices.

Proof. Let $t$ and $t^{\prime}$ be couple of given vertices. By $G$ is fuzzy complete, $N(t)=N\left(t^{\prime}\right)$. By $G$ is a fixed-vertex fuzzy graph, $\mu(t s)=\mu\left(t^{\prime} s\right)$, for all edges $t s, t^{\prime} s \in E$. Thus $t$ and $t^{\prime}$ are fuzzy twin vertices. $t$ and $t^{\prime}$ are arbitrary couple of vertices, hence every couple of vertices are fuzzy twin vertices. 
Theorem 3.16. Let $\mathcal{G}$ be a family of fuzzy graphs with common vertex set and $G \in \mathcal{G}$ is a fixed-vertex fuzzy graph such that it's fuzzy complete. Then simultaneously fuzzy metric number is $n-1$, simultaneously fuzzy metric dimension is $(n-1) \sigma(m)$ where $m$ is a given vertex. Every $(n-1)$-set is simultaneously fuzzy metric set for $\mathcal{G}$.

Proof. $G$ is fixed-vertex fuzzy graph and it's fuzzy complete. So by Proposition (3.15), we get every couple of vertices in fuzzy complete are fuzzy twin vertices. So every couple of vertices, by Theorem (3.14), aren't resolved.

Theorem 3.17. Let $\mathcal{G}$ be a family of fuzzy graphs with common vertex set and for every given couple of vertices, there's a $G \in \mathcal{G}$ such that in that, they're fuzzy twin vertices. Then simultaneously fuzzy metric number is $n-1$, simultaneously fuzzy metric dimension is $(n-1) \sigma(m)$ where $m$ is a given vertex. Every $(n-1)$-set is simultaneously fuzzy metric set for $\mathcal{G}$.

Theorem 3.18. Let $\mathcal{G}$ be a family of fuzzy graphs with common vertex set. If $\mathcal{G}$ contains three fixed-vertex fuzzy stars with different center, then simultaneously fuzzy metric number is $n-2$, simultaneously fuzzy metric dimension is $(n-2) \sigma(m)$ where $m$ is a given vertex. Every $(n-2)$-set is simultaneously fuzzy metric set for $\mathcal{G}$.

Proof. By Corollary (3.9), the result follows.

\section{References}

1. M. Akram, and G. Shahzadi, Operations on Single-Valued Neutrosophic Graphs, Journal of uncertain systems 11 (1) (2017) 1-26.

2. K. Atanassov, Intuitionistic fuzzy sets, Fuzzy Sets Syst. 20 (1986) 87-96.

3. S. Broumi, M. Talea, A. Bakali and F. Smarandache, Single-valued neutrosophic graphs, Journal of New Theory 10 (2016) 86-101.

4. N. Shah, and A. Hussain, Neutrosophic soft graphs, Neutrosophic Set and Systems 11 (2016) 31-44.

5. Henry Garrett, Big Sets Of Vertices, Preprints 2021, 2021060189 (doi: 10.20944/preprints202106.0189.v1).

6. Henry Garrett, Locating And Location Number, Preprints 2021, 2021060206 (doi: 10.20944/preprints202106.0206.v1).

7. Henry Garrett, Metric Dimensions Of Graphs, Preprints 2021, 2021060392 (doi: 10.20944/preprints202106.0392.v1).

8. Henry Garrett, New Graph Of Graph, Preprints 2021, 2021060323 (doi: 10.20944/preprints202106.0323.v1).

9. Henry Garrett, Numbers Based On Edges, Preprints 2021, 2021060315 (doi: 10.20944/preprints202106.0315.v1).

10. Henry Garrett, Matroid And Its Outlines, Preprints 2021, 2021060146 (doi: 10.20944/preprints202106.0146.v1).

11. Henry Garrett, Matroid And Its Relations, Preprints 2021, 2021060080 (doi: 10.20944/preprints202106.0080.v1).

12. A. Shannon and K.T. Atanassov, A first step to a theory of the intuitionistic fuzzy graphs, Proceeding of FUBEST (Lakov, D., Ed.) Sofia (1994) 59-61. 
13. F. Smarandache, A Unifying field in logics neutrosophy: Neutrosophic probability, set and logic, Rehoboth: American Research Press (1998).

14. H. Wang, F. Smarandache, Y. Zhang, and R. Sunderraman, Single-valued neutrosophic sets, Multispace and Multistructure 4 (2010) 410-413.

15. L. A. Zadeh, Fuzzy sets, Information and Control 8 (1965) 338-353. 